\title{
Mortar coupling for heterogeneous partial differential equations
}

\author{
Pablo Blanco ${ }^{1}$, Paola Gervasio ${ }^{2}$, and Alfio Quarteroni ${ }^{3}$
}

Submitted to the proceedings of 20th International Conference on Domain Decomposition

Methods, 7-11 February 2011, UC San Diego, California

\section{Introduction}

We are interested in the approximation of $2 \mathrm{D}$ elliptic equations with dominated advection and featuring boundary layers. In order to reduce the computational complexity, the domain is split into two subregions, the first one far from the layer, where we can neglect the viscosity effects, and the second one next to the layer. In the latter domain the original elliptic equation is solved, while in the former one, the pure convection equation obtained by the original one by dropping the diffusive term is approximated. The interface coupling is enforced by the non-conforming mortar method. We consider two different sets of interface conditions and we compare them for what concerns both computational efficiency and stability. One of the two sets of interface conditions turns out to be very effective, especially for very small viscosity when the mortar formulation of the original elliptic problem on the global domain can fail.

\section{The heterogeneous problem}

We consider an open bounded domain $\Omega \subset \mathbb{R}^{2}$ with Lipschitz boundary $\partial \Omega$, split into two open subsets $\Omega_{1}$ and $\Omega_{2}$ such that $\bar{\Omega}=\bar{\Omega}_{1} \cup \bar{\Omega}_{2}, \Omega_{1} \cap \Omega_{2}=\emptyset$. Then, we denote by $\Gamma=\partial \Omega_{1} \cap \partial \Omega_{2}$, the interface between the sub domains and we assume that $\Gamma$ is of class $C^{1,1}$. Given $f \in L^{2}(\Omega), b_{0} \in L^{\infty}(\Omega)$, $\nu \in L^{\infty}\left(\Omega_{2} \cup \Gamma\right)$ and $\mathbf{b} \in\left[W^{1, \infty}(\Omega)\right]^{2}$ satisfying the following inequalities:

${ }^{1}$ LNCC, Laboratório Nacional de Computação Científica, Av. Getúlio Vargas 333, Quitandinha, 25651-075 Petrópolis, RJ, Brazil. pjblanco@lncc.br . ${ }^{2}$ Department of Mathematics, University of Brescia, via Valotti, 9. 25133 Brescia, Italy. gervasio@ing.unibs.it . ${ }^{3} \mathrm{MOX}$, Department of Mathematics "F. Brioschi", Politecnico di Milano, via Bonardi, 9. 20133 Milano, Italy and SB-MATHICSE-CMCS École Polytechnique Fédérale de Lausanne CH1015 Lausanne, Switzerland. alfio.quarteroni@epfl.ch 


$$
\begin{gathered}
\exists \nu_{0} \in \mathbb{R} \text { such that } \nu(\mathbf{x}) \geq \nu_{0}>0, \forall \mathbf{x} \in \Omega_{2} \cup \Gamma, \\
\exists \sigma_{0} \in \mathbb{R} \text { such that } b_{0}(\mathbf{x})+\frac{1}{2} \operatorname{divb}(\mathbf{x}) \geq \sigma_{0}>0, \forall \mathbf{x} \in \Omega,
\end{gathered}
$$

we look for two functions $u_{1}$ and $u_{2}$ (defined in $\bar{\Omega}_{1}$ and $\bar{\Omega}_{2}$, respectively) solutions of the heterogeneous problem

$$
\begin{cases}\operatorname{div}\left(\mathbf{b} u_{1}\right)+b_{0} u_{1}=f & \text { in } \Omega_{1}, \\ \operatorname{div}\left(-\nu \nabla u_{2}+\mathbf{b} u_{2}\right)+b_{0} u_{2}=f & \text { in } \Omega_{2}, \\ u_{1}=0 & \text { on }\left(\partial \Omega_{1} \backslash \Gamma\right)^{i n} \\ u_{2}=0 & \text { on } \partial \Omega_{2} \backslash \Gamma\end{cases}
$$

and satisfying the interface conditions

$$
u_{1}=u_{2} \quad \text { on } \Gamma^{i n}, \quad \mathbf{b} \cdot \mathbf{n}_{\Gamma} u_{1}+\nu \frac{\partial u_{2}}{\partial \mathbf{n}_{\Gamma}}-\mathbf{b} \cdot \mathbf{n}_{\Gamma} u_{2}=0, \quad \text { on } \Gamma .
$$

$\mathbf{n}_{\Gamma}$ denotes the normal unit vector to $\Gamma$ oriented from $\Omega_{1}$ to $\Omega_{2}$, while for any non-empty subset $S \subseteq \partial \Omega_{1}, S^{i n}=\left\{\mathbf{x} \in S: \mathbf{b}(\mathbf{x}) \cdot \mathbf{n}_{1}(\mathbf{x})<0\right\}$ and $S^{\text {out }}=\left\{\mathbf{x} \in S: \mathbf{b}(\mathbf{x}) \cdot \mathbf{n}_{1}(\mathbf{x}) \geq 0\right\}$ are the inflow and the outflow parts of $S$, respectively.

Equations (2) (named IC1) express the continuity of the velocity field across the inflow part of the interface and the continuity of the fluxes across the whole interface. They can be equivalently expressed as (named IC2):

$$
u_{1}=u_{2}, \nu \frac{\partial u_{2}}{\partial \mathbf{n}_{\Gamma}}=0 \quad \text { on } \Gamma^{i n}, \quad-\mathbf{b} \cdot \mathbf{n}_{\Gamma} u_{1}=\nu \frac{\partial u_{2}}{\partial \mathbf{n}_{\Gamma}}-\mathbf{b} \cdot \mathbf{n}_{\Gamma} u_{2} \text { on } \Gamma^{\text {out }} .(3)
$$

Problem (1) with either interface conditions (2) or (3) is well-posed, see [5].

The heterogeneous problem (1), with either interface conditions IC1 or IC2, can formally be written as an interface problem by means of SteklovPoincaré operators (see, e.g., $[5,3]$ ). Let us define the trace spaces $\Lambda_{1}=$ $L_{\mathbf{b}}^{2}\left(\Gamma^{i n}\right)=\left\{v: \Gamma^{i n} \rightarrow \mathbb{R}: \sqrt{\left|\mathbf{b} \cdot \mathbf{n}_{\Gamma}\right|} v \in L^{2}\left(\Gamma^{i n}\right)\right\}$ and $\Lambda_{2}=H_{00}^{1 / 2}\left(\Gamma^{i n}\right)=$ $\left\{v: L^{2}\left(\Gamma^{i n}\right): \exists \tilde{v} \in H^{1 / 2}\left(\partial \Omega_{2}\right):\left.\tilde{v}\right|_{\Gamma^{i n}}=v,\left.\tilde{v}\right|_{\partial \Omega_{2} \backslash \Gamma^{i n}}=0\right\}$.

Solving (1),(2) is equivalent to seeking $\lambda_{k} \in \Lambda_{k}$ for $k=1,2$, such that

$$
\begin{cases}\mathcal{S}_{1} \lambda_{1}+\mathcal{S}_{2} \lambda_{2}=\chi_{1}+\chi_{2} & \text { in } \Lambda_{2}^{\prime}, \\ \lambda_{1}=\left.\lambda_{2}\right|_{\Gamma^{i n}} & \text { in } \Lambda_{2}\end{cases}
$$

where

$$
\mathcal{S}_{1} \lambda_{1}=-\mathbf{b} \cdot \mathbf{n}_{1} u_{1}^{\lambda_{1}}, \quad \mathcal{S}_{2} \lambda_{2}=\nu \frac{\partial u_{2}^{\lambda_{2}}}{\partial \mathbf{n}_{2}}-\mathbf{b} \cdot \mathbf{n}_{2} u_{2}^{\lambda_{2}}, \quad \text { on } \Gamma
$$

are the local Steklov-Poincaré operators, while $u_{1}^{\lambda_{1}}$ and $u_{2}^{\lambda}$ are the solution of

$$
\left\{\begin{array}{l}
\operatorname{div}\left(\mathbf{b} u_{1}^{\lambda_{1}}\right)+b_{0} u_{1}^{\lambda_{1}}=0 \text { in } \Omega_{1}, \\
u_{1}^{\lambda_{1}}=0 \text { on }\left(\partial \Omega_{1} \backslash \Gamma\right)^{i n}, \quad u_{1}^{\lambda_{1}}=\lambda \text { on } \Gamma^{i n}
\end{array}\right.
$$


and

$$
\left\{\begin{array}{l}
\operatorname{div}\left(-\nu \nabla u_{2}^{\lambda_{2}}+\mathbf{b} u_{2}^{\lambda_{2}}\right)+b_{0} u_{2}^{\lambda_{2}}=0 \text { in } \Omega_{2} \\
u_{2}^{\lambda_{2}}=0 \text { on } \partial \Omega_{2} \backslash \Gamma, \quad u_{2}^{\lambda_{2}}=\lambda_{2} \text { on } \Gamma
\end{array}\right.
$$

respectively. Finally,

$$
\chi_{1}=\mathbf{b} \cdot \mathbf{n}_{1} u_{1}^{f}, \quad \chi_{2}=-\nu \frac{\partial u_{2}^{f}}{\partial \mathbf{n}_{2}}+\mathbf{b} \cdot \mathbf{n}_{2} u_{2}^{f}=-\nu \frac{\partial u_{2}^{f}}{\partial \mathbf{n}_{2}},
$$

where $u_{1}^{f}$ and $u_{2}^{f}$ are the solutions of problems like (6) and (7), respectively, with null trace on the interface and external load $f$. Note that $\left.\chi_{1}\right|_{\Gamma^{\text {in }}}=0$.

If interface conditions IC2 are considered instead of IC1, the resulting Steklov-Poincaré equation reads: seek $\lambda_{k} \in \Lambda_{k}$, for $k=1,2$ such that

$$
\begin{cases}\mathcal{S}_{1}^{0} \lambda_{1}+\mathcal{S}_{2}^{0} \lambda_{2}=\chi_{1}+\chi_{2} & \text { in } \Lambda_{2}^{\prime} \\ \lambda_{1}=\left.\lambda_{2}\right|_{\Gamma^{\text {in }}} & \text { in } \Lambda_{2}\end{cases}
$$

where

$$
\mathcal{S}_{1}^{0} \lambda_{1}=\left\{\begin{array}{ll}
0 & \text { on } \Gamma^{\text {in }} \\
-\mathbf{b} \cdot \mathbf{n}_{1} u_{1}^{\lambda_{1}} & \text { on } \Gamma^{\text {out }},
\end{array} \mathcal{S}_{2}^{0} \lambda_{2}= \begin{cases}\nu \frac{\partial u_{2}^{\lambda_{2}}}{\partial \mathbf{n}_{2}} & \text { on } \Gamma^{\text {in }} \\
\nu \frac{\partial u_{2}^{\lambda_{2}}}{\partial \mathbf{n}_{2}}-\mathbf{b} \cdot \mathbf{n}_{2} u_{2}^{\lambda_{2}} & \text { on } \Gamma^{\text {out }} .\end{cases}\right.
$$

Remark 1. It is straightforward to prove that the operator $\mathcal{S}_{2}^{0}$ is always coercive on $\Lambda_{2}$, whereas $\mathcal{S}_{2}$ is coercive only if smallness assumption on $\mathbf{b}$ is assumed. If, e.g.,

$$
\|\mathbf{b}\|_{L^{\infty}(\Gamma)} \leq \varepsilon_{0}, \text { with } 0 \leq \varepsilon_{0} \leq 2 \min \left\{\nu_{0}, \sigma_{0}\right\} / C_{*}^{2},
$$

(where $C_{*}$ is the constant of the trace inequality $\|v\|_{L^{2}\left(\partial \Omega_{2}\right)} \leq C_{*}\|v\|_{H^{1}\left(\Omega_{2}\right)}$ ) is satisfied then $\mathcal{S}_{2}$ is coercive on $\Lambda_{2}$. For this reason, the solution of problem (4) may produce oscillations around $\Gamma^{i n}$ when advection dominates (i.e. the global Péclet number is large), as will be shown later in our numerical results.

\section{Mortar coupling for Spectral Element discretization}

The discretization of the differential equation within each sub domain is performed by the quadrilateral conforming Spectral Element Method (SEM). We refer to [4] for a detailed description of this method. For $k=1,2$, let $\mathcal{T}_{k}=\left\{T_{k, m}\right\}_{m=1}^{M_{k}}$ be a partition of the computational domain $\Omega_{k} \subset \mathbb{R}^{2}$. The SEM finite dimensional space on $\bar{\Omega}_{k}$ is denoted by $X_{k, \delta_{k}}$ and it is the set of functions in $C^{0}\left(\bar{\Omega}_{k}\right)$ whose restriction to $T_{k, m}$ is a polynomial of degree $N_{k}$ in each direction. $\delta_{k}$ is an abridged notation for "discrete", that accounts for 
the local geometric sizes $h_{k, m}$ of $T_{k, m}$ and the local polynomial degrees $N_{k}$ along each direction. Both geometric and polynomial conformity is guaranteed inside $\bar{\Omega}_{k}$.

The finite dimensional spaces in which we look for the SEM solution of either (4) or (9) are: $\Lambda_{1, \delta_{1}} \subset \Lambda_{1}$ and $\Lambda_{2, \delta_{2}} \subset \Lambda_{2}$. Their elements are globally continuous functions on $\Gamma^{i n}$ and $\Gamma$, respectively, and local polynomials of degree $N_{k}$ on each edge induced by the partition $\mathcal{T}_{k}$.

For $k=1,2$, we denote by $\mathcal{N}_{k, \Gamma}$ the set of nodes of $\mathcal{T}_{k} \cap \Gamma$ whose cardinality is $N_{k, \Gamma}$. Similar notations are used for the nodes lying on either $\Gamma^{\text {in }}$ or $\Gamma^{\text {out }}$.

The finite dimensional basis $\left\{\mu_{1}^{(i)}\right\}_{i=1}^{N_{1, \Gamma^{i n}}}$ of $\Lambda_{1, \delta_{1}}\left(\left\{\mu_{2}^{(i)}\right\}_{i=1}^{N_{2, \Gamma}}\right.$ of $\Lambda_{2, \delta_{2}}$, resp.) is composed by the characteristic Lagrange polynomials in $\Omega_{1}\left(\Omega_{2}\right.$, resp.) associated to the Legendre-Gauss-Lobatto (LGL) nodes of $\mathcal{N}_{1, \Gamma^{\text {in }}}\left(\mathcal{N}_{2, \Gamma}\right.$, resp.). Then we set $\left(S_{2, \delta_{2}}\right)_{i j}=\int_{\Gamma} \mathcal{S}_{2} \mu_{2}^{(j)} \mu_{2}^{(i)} d \Gamma$ for $i, j=0, \ldots, N_{2, \Gamma}$, and analogous notations are used to define matrices $S_{2, \delta_{2}}^{0}, S_{1, \delta_{1}}$ and $S_{1, \delta_{1}}^{0}$. Because of the high cost to compute integrals exactly, all integrals are approximated by Legendre-Gauss-Lobatto (LGL) quadrature rules.

We consider non-conforming couplings, i.e. we suppose that either the two partitions $\mathcal{T}_{1}$ and $\mathcal{T}_{2}$ do not share the same edges on $\Gamma$ and/or the polynomial degrees do not coincide in the hyperbolic domain $\Omega_{1}$ and in the elliptic one $\Omega_{2}$. We adopt mortar methods (see, e.g., [2]) to glue non-conforming discretization across $\Gamma$.

The endpoints of the edges of $\mathcal{T}_{1} \cap \Gamma^{i n}$ are denoted by $v_{1}^{(i)}$, for $i=$ $1, \ldots, N_{1, v} \cdot \tilde{\Lambda}_{1, \delta_{1}}$ is a suitable finite dimensional space of functions living on $\Gamma^{i n}$ and its basis functions $\psi_{l}$ are characterized by being $L^{2}$ functions on $\Gamma^{i n}$ and local polynomials of degree $N_{1}-2$ on each edge of $\mathcal{T}_{1} \cap \Gamma^{i n}$. Therefore, the dimension of $\tilde{\Lambda}_{1, \delta_{1}}$ is $N_{\tilde{\Lambda}_{1}}=N_{1, \Gamma^{i n}}-N_{1, v}$. By choosing $\Omega_{2}$ as the master domain and $\Omega_{1}$ as the slave, the continuity constraint $\lambda_{1}=\left.\lambda_{2}\right|_{\Gamma^{\text {in }}}$ is imposed weakly, i.e. by requiring that

$$
\int_{\Gamma^{\text {in }}}\left(\lambda_{1, \delta_{1}}-\lambda_{2, \delta_{2}}\right) \psi_{l} d \Gamma=0 \quad \forall \psi_{l} \in \tilde{\Lambda}_{1, \delta_{1}},
$$

jointly with the strong continuity at the nodes $v_{1}^{(i)}$ of $\mathcal{T}_{1} \cap \Gamma^{i n}$, for $i=$ $1, \ldots, N_{1, v}$. This leads us to define a new set of mortar functions in $\Lambda_{1, \delta_{1}}$, which are denoted by $\tilde{\mu}_{1}^{(k)}$ (for $k=1, \ldots, N_{2, \Gamma^{i n}}$ ) and satisfy the constraints:

$$
\begin{cases}\tilde{\mu}_{1}^{(k)}\left(v_{1}^{(i)}\right)=\mu_{2}^{(k)}\left(v_{1}^{(i)}\right), & i=1, \ldots, N_{1, v} \text { and } v_{1}^{(i)} \text { being endpoint } \\ \int_{\Gamma^{i n}}\left(\tilde{\mu}_{1}^{(k)}-\mu_{2}^{(k)}\right) \psi_{l} d \Gamma=0, l=1, \ldots, N_{\tilde{\Lambda}_{1}} \text { and for all } \psi_{l} \in \tilde{\Lambda}_{1, \delta_{1}} .\end{cases}
$$

Remark 2. We choose $\Omega_{2}$ as the master domain because the nature of the heterogeneous problem requires to work with the trace of the elliptic solution on the whole interface and with the trace of the hyperbolic one only on $\Gamma^{i n}$. 
Therefore it is more convenient to have the master trace at disposal on the whole $\Gamma$, instead of on a part of it.

The matrix form of system (13) reads

$$
P \Xi=\Phi,
$$

where $\Xi=\left[\xi_{j k}\right] \in \mathbb{R}^{N_{1, \Gamma^{i n}} \times N_{2, \Gamma^{i n}}}$ is defined by the relations

$$
\tilde{\mu}_{1}^{(k)}=\sum_{j=1}^{N_{1, \Gamma^{i n}}} \xi_{j k} \mu_{1}^{(j)}, \quad k=1, \ldots, N_{2, \Gamma^{i n}},
$$

while $P \in \mathbb{R}^{N_{1, \Gamma^{i n}} \times N_{1, \Gamma^{i n}}}$ and $\Phi \in \mathbb{R}^{N_{1, \Gamma^{i n}} \times N_{2, \Gamma^{i n}}}$, are defined starting from (13). The matrix $P$ is non-singular in view of the inf-sup condition for $\mathbb{Q}_{N}-$ $\mathbb{Q}_{N-2}([2])$. Once the discretization in $\Omega_{1}$ and $\Omega_{2}$ has been chosen, the matrix $\Xi$ can be explicitly computed by solving (14).

The matrix $\Xi$ enforces the gluing between degrees of freedom defined on $\mathcal{N}_{2, \Gamma^{i n}}$ and $\mathcal{N}_{1, \Gamma^{i n}}$. Therefore, Steklov-Poincaré equations (4) and (9) can be written in a nonconforming setting, by the use of matrix $\Xi$.

On $\Gamma^{\text {out }}$ no continuity constraint, neither strong nor weak, is imposed, since the continuity of fluxes is a natural consequence of the interface equation. Nevertheless, on $\Gamma^{\text {out }}$ we have to compute integrals of basis functions associated to two different meshes. To this aim we introduce the matrix $Q \in \mathbb{R}^{N_{2, \Gamma^{\text {out }}} \times N_{1, \Gamma^{\text {out }}}}$ for the evaluations of functions of $\Lambda_{1, \delta_{1}}$ at the nodes of $\mathcal{T}_{2} \cap \Gamma$, and the matrix $D=M_{2, \delta_{2}}^{\text {out }} Q\left(M_{1, \delta_{1}}^{\text {out }}\right)^{-1}$, where $M_{k, \delta_{k}}^{\text {out }}$ are the mass matrices induced by the LGL quadrature formulas on $\Gamma^{\text {out }}$, for $k=1,2$.

The nonconforming finite dimensional counterpart of (4) reads: find $\lambda_{k, \delta_{k}} \in$ $\Lambda_{k, \delta_{k}}$ for $k=1,2$, such that

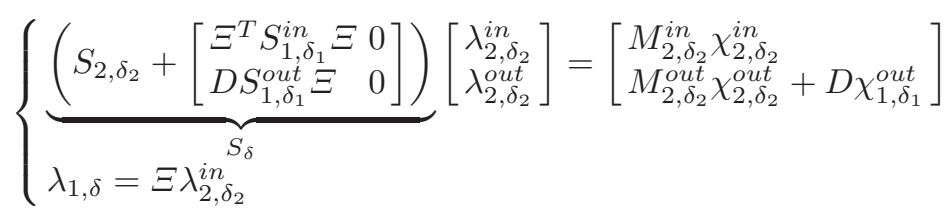

whereas that of (9) becomes: find $\lambda_{k, \delta_{k}} \in \Lambda_{k, \delta_{k}}$ for $k=1,2$, such that

$$
\left\{\begin{array}{l}
\underbrace{\left(S_{2, \delta_{2}}^{0}+\left[\begin{array}{ll}
0 & 0 \\
D S_{1, \delta_{1}}^{\text {out }} \Xi & 0
\end{array}\right]\right)}_{S_{\delta}^{0}}\left[\begin{array}{l}
\lambda_{2, \delta_{2}}^{\text {in }} \\
\lambda_{2, \delta_{2}}^{\text {out }}
\end{array}\right]=\left[\begin{array}{l}
M_{2, \delta_{2}}^{\text {in }} \chi_{2, \delta_{2}}^{\text {in }} \\
M_{2, \delta_{2}}^{\text {out }} \chi_{2, \delta_{2}}^{\text {out }}+D \chi_{1, \delta_{1}}^{\text {out }}
\end{array}\right] \\
\lambda_{1, \delta}=\Xi \lambda_{2, \delta_{2}}^{\text {in }} .
\end{array}\right.
$$

The upper scripts in and out denote the restriction to $\Gamma^{\text {in }}$ and $\Gamma^{\text {out }}$, resp.

The numerical solutions of these linear systems is carried out by preconditioned Bi-CGStab iterations (see, [6]). 
When conforming discretization is used across the interface (i.e. $\delta_{1}=\delta_{2}$ ), matrix $\Xi$ reduces to the identity matrix. In this situation, it is well known (see, e.g. [5]) that $S_{2, \delta_{2}}^{0}$ is an optimal preconditioner for the matrix $S_{\delta}^{0}$, i.e. $\exists C_{0}>0$ independent of $\delta$ such that its spectral condition number $\mathcal{K}\left(\left(S_{2, \delta_{2}}^{0}\right)^{-1} S_{\delta}^{0}\right)$ is bounded by $C_{0}$. When $\delta_{1}=\delta_{2}, S_{2, \delta_{2}}^{0}$ is an optimal preconditioner also for $S_{\delta}$ (see [3]), i.e. there exists $C_{1}>0$ independent of $\delta$ such that $\mathcal{K}\left(\left(S_{2, \delta_{2}}^{0}\right)^{-1} S_{\delta}\right) \leq C_{1}$, and numerical results show that $C_{0} \leq C_{1}$.

We extend here the use of the preconditioner $S_{2, \delta}^{0}$ to the non-conforming case.

\section{Numerical results}

Test case: the computational domain $\Omega=(-1,1)^{2}$ is split in $\Omega_{1}=(-1,0.8) \times$ $(-1,1)$ and $\Omega_{2}=(0.8,1) \times(-1,1)$. The interface is $\Gamma=\{0.8\} \times(-1,1)$. The data of the problem are: $\mathbf{b}=[5 y, 1-x]^{t}, b_{0}=1, f=1$ and the inflow interface is $\Gamma^{i n}=\{0.8\} \times(-1,0)$. The imposed Dirichlet boundary conditions are: $u_{1}=1$ on $((-1,0.8) \times\{-1\}) \cup(\{-1\} \times(0,1)), u_{2}=0$ on $\{1\} \times(-1,1)$, $u_{2}=1$ on $(0.8,1) \times\{-1\}$, while the homogeneous Neumann condition $\frac{\partial u_{2}}{\partial \mathbf{n}_{2}}=0$ is imposed on $(0.8,1) \times\{1\}$.

Because of the presence of a boundary layer near the right vertical side, the mesh is refined there (without losing the conformity inside $\Omega_{2}$ ) to prevent the numerical solution to be affected by spurious oscillations.

In Fig. 1 the number of Preconditioned Bi-CGStab (PBi-CGStab) iterations (with preconditioner $S_{2, \delta_{2}}$ ) required to reduce the relative norm of the residual of 12 orders of magnitude is plotted versus the polynomial degrees $N_{1}$ and $N_{2}$ of the mortar discretization. These results refer to $\nu=10^{-2}$ and show that the Steklov-Poincaré formulation (9) performs better than (4). The analysis of this and other test cases leads us to conjecture that $\mathcal{K}\left(\left(S_{2, \delta_{2}}^{0}\right)^{-1} S_{\delta}^{0}\right) \leq C_{0}$ still holds for non-conforming coupling $\left(\delta_{1} \neq \delta_{2}\right)$, while

$$
\mathcal{K}\left(\left(S_{2, \delta_{2}}^{0}\right)^{-1} S_{\delta}\right) \simeq C_{1} \mathcal{K}\left(\Xi \Xi^{T}\right) \simeq C_{1} \begin{cases}\left(N_{2}-N_{1}+1\right)^{3 / 2} & \text { if } N_{1}<N_{2} \\ C_{2} & \text { if } N_{1} \geq N_{2},\end{cases}
$$

where $C_{1}$ is the constant defined in the previous section, and $C_{2}$ is another positive constant independent of $\delta$.

Therefore, formulation (17) corresponding to IC2 is optimally preconditioned by $S_{2, \delta_{2}}^{0}$ and it is better than (16) (corresponding to IC1) for what concerns the computational efficiency.

Moreover, when the viscosity vanishes (see Table 1), the performance of the SP0 approach (17) does not downgrade, as the number of PBi-CGStab iterations keeps bounded: 3 or 4 iterations are enough to satisfy the stopping test independently of both viscosity and discretization parameters. 

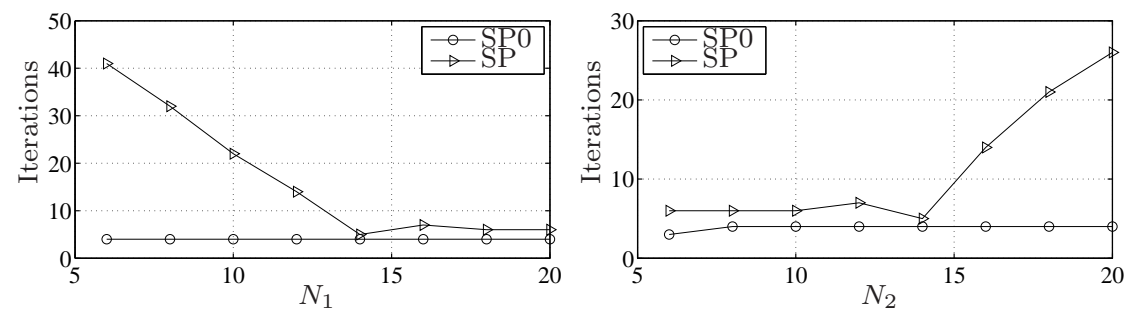

Fig. 1 Preconditioned Bi-CGStab iterations. The viscosity is $\nu=10^{-2}$. At left, $N_{2}=14$ is fixed, at right, $N_{1}=14$ is fixed. $4 \times 4$ equal spectral elements are taken in each $\Omega_{k}$

On the contrary, the number of PBi-CGStab iterations required by SP approach (16) noticeably grows up when $\nu \rightarrow 0$ and behaves like $\left(N_{2}-N_{1}+\right.$ $1)^{3 / 4}$ when $N_{1}<N_{2}$, in agreement with (18).

The large number of PBi-CGStab iterations required by SP is due to the presence of instabilities across $\Gamma^{\text {in }}$ which develop when advection dominates and the larger $N_{2}-N_{1}$ is, the more they are pronounced.

We verified that the same instability occurs when mortar methods are applied to solve the pure elliptic-elliptic couplings with dominated advection and interface condition $\nu \frac{\partial u_{1}}{\partial \mathbf{n}_{\Gamma}}-\mathbf{b} \cdot \mathbf{n}_{\Gamma} u_{1}=\nu \frac{\partial u_{2}}{\partial \mathbf{n}_{\Gamma}}-\mathbf{b} \cdot \mathbf{n}_{\Gamma} u_{2}$ on the whole interface $\Gamma$. Indeed, the local Steklov-Poincaré operators associated to the latter interface condition behaves like operator $\mathcal{S}_{2}$ introduced in (5), and they can lose the coercivity when $\|\mathbf{b}\|_{L^{\infty}(\Omega)}$ is large. This is the subject of a work in progress. (See also[1].)

In conclusion, the heterogeneous approach (1) with interface conditions IC2 and non-conforming mortar coupling turns out to be the most efficient and accurate one for vanishing viscosity and it is also a valid way to overcome instabilities arising from the mortar discretization of elliptic equations with dominated advection.

In Fig. 2 the heterogeneous solutions obtained by solving both (17) and (16) with $\nu=10^{-4}, N_{1}=8$ and $N_{2}=24$ are shown. The elliptic solution $u_{2}$ provided by (16) (Fig. 2, right) exhibits non-trivial oscillations, while that provided by (17) (Fig. 2, left) does not.

Table 1 PBi-CGStab iterations to solve systems SP0 (17) and SP (16) with $P=S_{2, \delta_{2}}^{0}$ versus the viscosity. At left, $N_{1}=8$, at right, $N_{1}=20, N_{2}=24.4 \times 4$ equal spectral elements are taken in each $\Omega_{k} . N_{2}=64$ along $x$-direction in the elements next to the layer

\begin{tabular}{|c|c|c|c|c|c|c|c|c|c|}
\hline$\nu$ & $10^{-1}$ & $10^{-2}$ & $10^{-3}$ & $10^{-4}$ & $\nu$ & $10^{-1}$ & $10^{-2}$ & $10^{-3}$ & $10^{-4}$ \\
\hline SP0 & 3 & 4 & 3 & 3 & $\overline{\mathrm{SP} 0}$ & 3 & 3 & 3 & 4 \\
\hline SP & 10 & 45 & 262 & 587 & SP & 7 & 17 & 35 & 86 \\
\hline
\end{tabular}



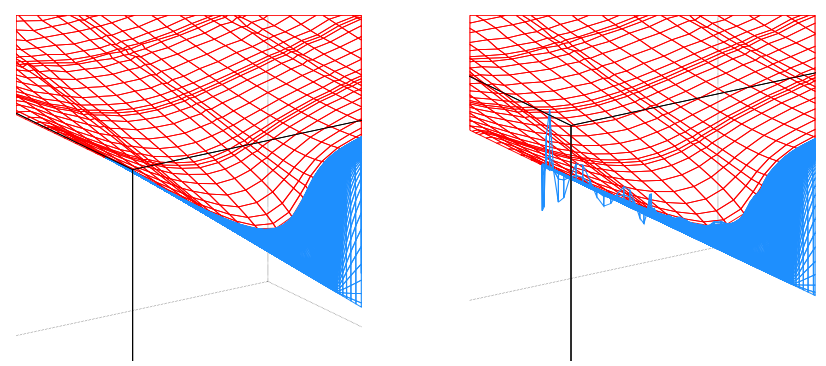

Fig. 2 Zoom on the numerical solution for $\nu=10^{-3}$ and: (9) (left), (4) (right) with $N_{1}=8$ and $N_{2}=24$. The elliptic solution $u_{2}$ is in front, while the hyperbolic one $u_{1}$ is behind.

\section{References}

[1] Y. Achdou. The mortar element method for convection diffusion problems. C.R. Acad. Sci. Paris. Sér. I Math., 321:117-123, 1995.

[2] C. Bernardi, Y. Maday, and A.T. Patera. A new nonconforming approach to domain decomposition: the mortar element method. In Nonlinear partial differential equations and their applications. Collège de France Seminar, Vol. XI (Paris, 1989-1991), volume 299 of Pitman Res. Notes Math. Ser., pages 13-51. Longman Sci. Tech., Harlow, 1994.

[3] P.J. Blanco, P. Gervasio, and A. Quarteroni. Extended variational formulation for heterogeneous partial differential equations. Technical report, EPFL-REPORT-165290, EPFL, Lausanne, Switzerland, 2011. Accepted for publication in Computational Methods in Applied Mathematics.

[4] C. Canuto, M. Y. Hussaini, A. Quarteroni, and T. A. Zang. Spectral Methods. Evolution to Complex Geometries and Applications to Fluid Dynamics. Springer, Heidelberg, 2007.

[5] F. Gastaldi, A. Quarteroni, and G. Sacchi Landriani. On the coupling of two dimensional hyperbolic and elliptic equations: analytical and numerical approach. In J.Périeaux T.F.Chan, R.Glowinski and O.B.Widlund, editors, Third International Symposium on Domain Decomposition Methods for Partial Differential Equations, pages 22-63, Philadelphia, 1990. SIAM.

[6] H.A. van der Vorst. Iterative Krylov methods for large linear systems, volume 13 of Cambridge Monographs on Applied and Computational Mathematics. Cambridge University Press, Cambridge, 2003. 